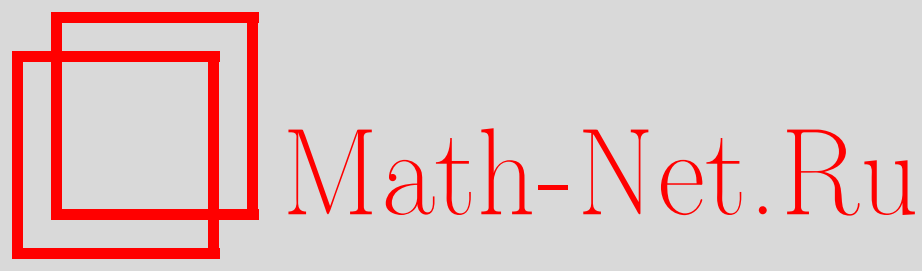

О. Н. Хакимов, p-Адические меры Гиббса для модели твердых сфер с тремя состояниями на дереве Кэли, TMФ, 2013, том 177, номер 1, 68-82

DOI: https://doi.org/10.4213/tmf8603

Использование Общероссийского математического портала Math-Net.Ru подразумевает, что вы прочитали и согласны с пользовательским соглашением http://www.mathnet.ru/rus/agreement

Параметры загрузки:

IP: 3.82 .47 .9

26 апреля 2023 г., 18:07:59

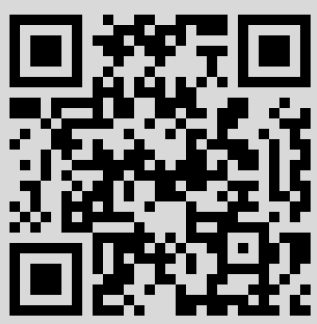


Том 177, № 1

октябрь, 2013

(C) 2013 г.

\author{
О.Н. Хакимов*
}

\title{
p-АДИЧЕСКИЕ МЕРЫ ГИББСА \\ ДЛЯ МОДЕЛИ ТВЕРДЫХ СФЕР С ТРЕМЯ СОСТОЯНИЯМИ НА ДЕРЕВЕ КЭЛИ
}

\begin{abstract}
Изучаются $p$-адические модели твердых сфер с тремя состояниями на дереве Кэли. Известно, что существуют четыре типа таких моделей. Найдены условия, которые необходимо наложить на порядок $k$ дерева Кэли и простое число $p$, чтобы существовала трансляционно-инвариантная $p$-адическая мера Гиббса.
\end{abstract}

Ключевые слова: дерево Кэли, конфигурация, мера Гиббса, $G$-модель твердых сфер, трансляционно-инвариантная мера, $p$-адические числа.

DOI: $10.4213 / \operatorname{tmf} 8536$

\section{1. ВВЕДЕНИЕ}

В работах [1], [2] были изучены вещественные меры Гиббса для модели твердых сфер (ТС) с тремя состояниями на дереве Кэли порядка $k \geqslant 1$. В настоящей работе мы изучаем p-адический аналог этой модели.

Описание предельных мер Гиббса для данного гамильтониана является одной из основных задач в теории мер Гиббса. Полный анализ множества таких мер является весьма трудоемким. По этой причине большая часть работ по этой тематике посвящена изучению мер Гиббса на дереве Кэли [1], [3]--[7].

Известно [8]-[10], что $p$-адические модели в физике нельзя описать, используя обычную теорию вероятностей. В книге [8] на основе теории неархимедовых мер [11] была сформулирована абстрактная $p$-адическая теория вероятностей. Вероятностные процессы над полем $p$-адических чисел были рассмотрены во многих работах (см., например, [12]-[15]). Неархимедов аналог теоремы Колмогорова был доказан в работе [16]. В работе [17] рассмотрены плодородные модели ТС, соответствующие графам типа петля, свисток, жезл и ключ. В работе [1] изучались трансляционноинвариантные и периодические меры Гиббса для модели ТС в случае графа типа ключ на дереве Кэли и доказана единственность трансляционно-инвариантной меры

* Институт математики и информационных технологий АН РУз, Ташкент, Узбекистан. E-mail: hakimovo@mail.ru 
Гиббса для любого положительного параметра активности. В работе [2] рассматривались трансляционно-инвариантные и периодические меры Гиббса для модели ТС в случаях графов типа свисток, жезл, петля и показано, что в случае графа типа петля из положительности активности не следует единственность трансляционноинвариантных мер.

Трансляционно-инвариантные $p$-адические меры Гиббса для случая графа типа петля изучались в работе [18]. В настоящей работе мы проведем анализ трансляционно-инвариантных $p$-адических мер Гиббса для модели ТС на дереве Кэли в случаях графов типа свисток, жезл и ключ.

\section{2. ОПРЕДЕЛЕНИЯ И ОСНОВНЫЕ ПОЛОЖЕНИЯ}

2.1. $p$-Адические числа и меры. Каждое рациональное число $x \neq 0$ может быть представлено в виде

$$
x=p^{r} \frac{n}{m}, \quad r, n, m \in \mathbb{Z}, \quad m>0,
$$

где $p$ - фиксированное простое число, числа $m$ и $n$ взаимно простые (наибольший общий делитель $(n, m)$ равен единице), причем $m$ и $n$ не делятся на $p$. Далее, $p$-адическая норма $|x|_{p}$ определяется по формуле

$$
|x|_{p}= \begin{cases}p^{-r}, & \text { если } x \neq 0, \\ 0, & \text { если } x=0 .\end{cases}
$$

Она удовлетворяет сильному неравенству треугольника $|x+y|_{p} \leqslant \max \left\{|x|_{p},|y|_{p}\right\}$, что показывает неархимедовость нормы. Из этого свойства непосредственно вытекают следующие:

1) если $|x|_{p} \neq|y|_{p}$, то $|x-y|_{p}=\max \left\{|x|_{p},|y|_{p}\right\}$;

2) если $|x|_{p}=|y|_{p}$, то $|x-y|_{p} \leqslant|x|_{p}$.

Для каждого простого $p$ пополнение поля рациональных чисел $\mathbb{Q}$ по $p$-адической норме приводит к полю $\mathbb{Q}_{p}$ р-адических чисел [19]. Начав с поля рациональных чисел $\mathbb{Q}$, мы можем получить либо поле вещественных чисел $\mathbb{R}$, либо одно из полей $p$-адических чисел $\mathbb{Q}_{p}$ (теорема Островского).

Каждое $p$-адическое число $x \neq 0$ имеет единственное каноническое разложение

$$
x=p^{\gamma(x)}\left(x_{0}+x_{1} p+x_{2} p^{2}+\cdots\right),
$$

где $\gamma=\gamma(x) \in \mathbb{Z}$ и $x_{j}$ - целые числа, $x_{0}>0,0 \leqslant x_{j} \leqslant p-1, j=1,2, \ldots$ [10], [19], [20]. В этом случае $|x|_{p}=p^{-\gamma(x)}$.

Следующая теорема доказана в работе [10].

ТеОрема 1. Уравнение $x^{2}=a$ при

$$
0 \neq a=p^{\gamma(a)}\left(a_{0}+a_{1} p+a_{2} p^{2}+\cdots\right), \quad a_{0}>0, \quad 0 \leqslant a_{j} \leqslant p-1, \quad j=1,2, \ldots,
$$

имеет решение $x \in \mathbb{Q}_{p}$ тогда и только тогда, когда выполняются следующие условия: число $\gamma(a)$ четное; уравнение $y^{2}=a_{0}(\bmod p)$ разрешимо, если $p \neq 2 ;$ равенство $a_{1}=a_{2}=0$ справедливо, если $p=2$. 
Множество $\mathbb{Z}_{p}=\left\{x \in \mathbb{Q}_{p}:|x|_{p} \leqslant 1\right\}$ называется множеством цельх $p$-адических чисел.

Следующая теорема известна как лемма Гензеля [19].

Теорема 2. Пусть $F(x)=c_{0}+c_{1} x+\cdots+c_{n} x^{n}-$ многочлен с иелыми р-адическими коэффициентами, а $F^{\prime}(x)=c_{1}+2 c_{2} x+\cdots+n c_{n} x^{n-1}-$ его производная. Предположим, что $a_{0}$ - целое р-адическое число такое, что $F\left(a_{0}\right)=0(\bmod p)$, но $F^{\prime}\left(a_{0}\right) \neq 0(\bmod p)$. Тогда существует единственное иелое р-адическое число а maкое, что $F(a)=0$ u $a \equiv a_{0}(\bmod p)$.

Для $a \in \mathbb{Q}_{p}$ и $r>0$ положим $B(a, r)=\left\{x \in \mathbb{Q}_{p}:|x-a|_{p}<r\right\} . \quad p$-Адический логарифм определяется как ряд

$$
\log _{p}(x)=\log _{p}(1+(x-1))=\sum_{n=1}^{\infty}(-1)^{n+1} \frac{(x-1)^{n}}{n},
$$

который сходится для $x \in B(1,1) ;$-адическая экспонента определяется как ряд

$$
\exp _{p}(x)=\sum_{n=0}^{\infty} \frac{x^{n}}{n !}
$$

который сходится для $x \in B\left(0, p^{-1 /(p-1)}\right)$.

ЛЕмма 1. Пусть $x \in B\left(0, p^{-1 /(p-1)}\right)$. Тогда

$$
\begin{gathered}
\left|\exp _{p}(x)\right|_{p}=1, \quad\left|\exp _{p}(x)-1\right|_{p}=|x|_{p}, \quad\left|\log _{p}(1+x)\right|_{p}=|x|_{p} \\
\log _{p}\left(\exp _{p}(x)\right)=x, \quad \exp _{p}\left(\log _{p}(1+x)\right)=1+x
\end{gathered}
$$

Более подробно об основах $p$-адического анализа и $p$-адической математической физики можно прочитать в книгах [10], [19], [20].

Пусть $(X, \mathcal{B})$ - измеримое пространство, где $\mathcal{B}$ - алгебра подмножеств в $X$. Функция $\mu: \mathcal{B} \rightarrow \mathbb{Q}_{p}$ называется $p$-адической мерой, если для любого набора множеств $A_{1}, \ldots, A_{n} \in \mathcal{B}$ таких, что $A_{i} \cap A_{j}=\varnothing$ при $i \neq j$, имеет место равенство

$$
\mu\left(\bigcup_{j=1}^{n} A_{j}\right)=\sum_{j=1}^{n} \mu\left(A_{j}\right)
$$

р-адическая мера называется вероятностной [16], если $\mu(X)=1$.

2.2. Дерево Кэли. Дерево Кэли $\Gamma^{k}=(V, L)$ порядка $k \geqslant 1$ есть бесконечное дерево (граф без циклов), из каждой вершины которого выходит ровно $k+1$ ребер. Здесь $V$ - множество вершин и $L$ - множество ребер. Две вершины $x$ и $y$ называются ближайшими соседями, если существует ребро $l \in L$, соединяющее их (что обозначается как $l=\langle x, y\rangle)$. Расстояние $d(x, y)$ равно количеству ребер, составляющих кратчайший путь, который соединяет $x$ и $y$.

Пусть $x^{0} \in V$ - фиксированная точка. Введем обозначения

$$
\begin{gathered}
W_{n}=\left\{x \in V: d\left(x, x^{0}\right)=n\right\}, \quad V_{n}=\bigcup_{k=1}^{n} W_{k}, \\
S(x)=\left\{y \in W_{n+1}: d(x, y)=1\right\} \text { для } x \in W_{n} .
\end{gathered}
$$


2.3. $G$-модель ТС. Мы рассмотрим модель ТС с тремя состояниями на дереве Кэли. В этой модели каждой вершине $x \in V$ ставится в соответствие одно из значений $\sigma(x) \in\{0,1,2\}$. Если $\sigma(x) \in\{1,2\}$, то это означает, что вершина $x \in V$ занята, а $\sigma(x)=0$ означает, что вершина $x \in V$ вакантна. Конфигурация $\sigma=\{\sigma(x), x \in V\}$ на дереве Кэли есть функция из $V$ в $\{0,1,2\}$. Конфигурации на множествах $V_{n}$ и $W_{n}$ определяются аналогично.

Рассмотрим четыре типа плодородных графов [17] с множеством ребер следующего вида:

$\begin{array}{rllll}\text { свисток: } & \{0,0\},\{0,1\},\{1,2\} ; \\ \text { ключ: } & \{0,0\},\{0,1\},\{0,2\},\{1,1\} ; \\ \text { жезл: } & \{0,1\},\{0,2\},\{1,1\},\{2,2\} ; \\ \text { петля: } & \{0,0\},\{0,1\},\{0,2\},\{1,1\},\{2,2\} .\end{array}$

Пусть $O=\{$ свисток, ключ, жезл, петля\}, $G \in O$. Конфигурация $\sigma$ называется $G$-допустимой на дереве Кэли, если $\{\sigma(x), \sigma(y)\}$ является ребром графа $G$ для любой пары ближайших соседей $x$ и $y$ в $V$. Обозначим через $\Omega^{G}$ множество всех $G$-допустимых конфигураций на дереве Кэли.

Для данного графа $G$ и фиксированного параметра $\lambda=\left(\lambda_{0}, \lambda_{1}, \lambda_{2}\right) \in \mathbb{Q}_{p}^{3}$ определим $p$-адический гамильтониан $G$-модели ТС как

$$
H_{G}^{\lambda}(\sigma)=\sum_{x \in V} \log _{p} \lambda_{\sigma(x)}, \quad \sigma \in \Omega^{G} .
$$

2.4. Построение $p$-адической меры Гиббса. Построим $p$-адическую меру Гиббса для $G$-модели ТС. Так как в определении $p$-адической меры Гиббса используется $p$-адическая экспонента, все соответствующие величины должны принадлежать множеству

$$
\mathcal{E}_{p}=\left\{x \in \mathbb{Q}_{p}:|x|_{p}=1,|x-1|_{p}<p^{-1 /(p-1)}\right\} .
$$

Как и в классическом случае, мы рассмотрим специальный класс мер Гиббса.

Для $\sigma_{n} \in \Omega_{V_{n}}^{G}$ обозначим через \# $\sigma_{n}$ число занятых вершин в конфигурации $\sigma_{n}$ :

$$
\# \sigma_{n}=\sum_{x \in V_{n}} \mathbf{1}\left(\sigma_{n}(x) \geqslant 1\right)
$$

Пусть $z: x \rightarrow z_{x}=\left(z_{0, x}, z_{1, x}, z_{2, x}\right) \in \mathcal{E}_{p}^{3}$ - векторнозначная функция на $V$. Рассмотрим случай $\lambda_{0}=1$ и $\lambda_{1}=\lambda_{2}=\lambda$. Для $\lambda \in \mathcal{E}_{p}$ рассмотрим $p$-адическое вероятностное распределение $\mu^{(n)}$ на множестве $\Omega_{V_{n}}^{G}$, которое определяется как

$$
\mu^{(n)}\left(\sigma_{n}\right)=Z_{n}^{-1} \lambda^{\# \sigma_{n}} \prod_{x \in W_{n}} z_{\sigma(x), x}, \quad n=1,2, \ldots
$$

где $Z_{n}$ - нормировочная постоянная,

$$
Z_{n}=\sum_{\tilde{\sigma}_{n} \in \Omega_{V_{n}}} \lambda^{\# \tilde{\sigma}_{n}} \prod_{x \in W_{n}} z_{\tilde{\sigma}(x), x} .
$$


Говорят, что $p$-адическое вероятностное распределение $\mu^{(n)}$ является согласованным, если для всех $n \geqslant 1$ и $\sigma_{n-1} \in \Omega_{V_{n-1}}^{G}$

$$
\sum_{\omega_{n} \in \Omega_{W_{n}}} \mu^{(n)}\left(\sigma_{n-1} \vee \omega_{n}\right) \mathbf{1}\left(\sigma_{n-1} \vee \omega_{n} \in \Omega_{V_{n}}^{G}\right)=\mu^{(n-1)}\left(\sigma_{n-1}\right)
$$

В этом случае в силу теоремы Колмогорова [16] существует единственная мера $\mu$ на множестве $\Omega^{G}$ такая, что $\mu\left(\left\{\left.\sigma\right|_{V_{n}}=\sigma_{n}\right\}\right)=\mu^{(n)}\left(\sigma_{n}\right)$ для всех $n$ и $\sigma_{n} \in \Omega_{V_{n}}^{G}$.

ОПРЕДЕлЕниЕ 1. Мера $\mu^{(n)}$, определенная в (1) и удовлетворяющая условию (2), называется $p$-адической $G$-TC-мерой Гиббса, соответствующей функции $z: x \rightarrow z_{x}$, $x \in V \backslash\left\{x^{0}\right\}$.

Пусть $L(G)$ - множество ребер графа $G$. Через $A \equiv A^{G}=\left(a_{i j}\right)_{i, j=0,1,2}$ обозначим матрицу смежности графа $G$, т. е.

$$
a_{i j} \equiv a_{i j}^{G}= \begin{cases}1, & \text { если }\{i, j\} \in L(G), \\ 0, & \text { если }\{i, j\} \notin L(G) .\end{cases}
$$

Следующая теорема [2] дает условие для $z_{x}$, гарантирующее согласованность распределения $\mu^{(n)}$.

Теорема 3. Вероятностное распределение $\mu^{(n)}, n=1,2, \ldots$, заданное формулой (1), согласованно тогда и только тогда, когда для любого $x \in V$ имеют место следующие равенства:

$$
\begin{aligned}
& z_{1, x}^{\prime}=\lambda \prod_{y \in S(x)} \frac{a_{10}+a_{11} z_{1, y}^{\prime}+a_{12} z_{2, y}^{\prime}}{a_{00}+a_{01} z_{1, y}^{\prime}+a_{02} z_{2, y}^{\prime}}, \\
& z_{2, x}^{\prime}=\lambda \prod_{y \in S(x)} \frac{a_{20}+a_{21} z_{1, y}^{\prime}+a_{22} z_{2, y}^{\prime}}{a_{00}+a_{01} z_{1, y}^{\prime}+a_{02} z_{2, y}^{\prime}},
\end{aligned}
$$

где $z_{i, x}^{\prime}=\lambda z_{i, x} / z_{0, x} \in \mathcal{E}_{p}, i=1,2$.

\section{3. ТРАНСЛЯЦИОННО-ИНВАРИАНТНАЯ МЕРА ГИББСА}

Предположим, что $z_{0, x} \equiv 1$ и $z_{i, x}=z_{i, x}^{\prime} \in \mathcal{E}_{p}, i=1,2$. Тогда для любой функции $z: x \in V \rightarrow z_{x}=\left(z_{1, x}, z_{2, x}\right) \in \mathcal{E}_{p}^{2}$ такой, что справедлива система уравнений (3), существует единственная $p$-адическая $G$-TC-мера Гиббса. Решение вида $z_{x}=\left(z_{1}, z_{2}\right) \in \mathcal{E}_{p}^{2}$ при $x \neq x_{0}$ системы уравнений (3) называется трансляиионно-инвариантным. Соответствующая $p$-адическая мера Гиббса трансляционно-инвариантного решения системы уравнений (3) называется трансляционно-инвариантной мерой Гиббса.

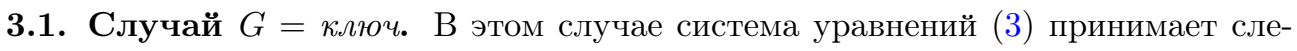
дующий вид:

$$
z_{1, x}=\lambda \prod_{y \in S(x)} \frac{1+z_{1, y}}{1+z_{1, y}+z_{2, y}}, \quad z_{2, x}=\lambda \prod_{y \in S(x)} \frac{1}{1+z_{1, y}+z_{2, y}} .
$$

Мы будем использовать следующую лемму [21]. 
ЛЕмма 2. Если $a_{i} \in \mathcal{E}_{p}$ для любого $i=1, \ldots, m$, mо

$$
\prod_{i=1}^{m} a_{i} \in \mathcal{E}_{p}
$$

Теорема 4. Если наибольший общий делитель $\left(2^{k}-1,3^{k}-1\right)$ не делится на $p$, то система уравнений (4) не имеет решений на множестве $\mathcal{E}_{p}^{2}$.

ДоказАтельство. Пусть $z_{x} \in \mathcal{E}_{p}$ - решение системы $(4), x \in V$. Тогда из уравнений (4) получим

$$
\begin{aligned}
\left|z_{1, x}\right|_{p} & =\left|\lambda \prod_{y \in S(x)} \frac{1+z_{1, y}}{1+z_{1, y}+z_{2, y}}\right|_{p}=\prod_{y \in S(x)} \frac{\left|1+z_{1, y}\right|_{p}}{\left|1+z_{1, y}+z_{2, y}\right|_{p}}= \\
& =\prod_{y \in S(x)} \frac{\left|2+z_{1, y}-1\right|_{p}}{\left|3+z_{1, y}-1+z_{2, y}-1\right|_{p}} \begin{cases}\leqslant 2^{-k}, & \text { если } p=2, \\
\geqslant 3^{k}, & \text { если } p=3, \\
=1, & \text { если } p \neq 2,3 ;\end{cases} \\
\left|z_{2, x}\right|_{p} & =\prod_{y \in S(x)} \frac{1}{\left|3+z_{1, y}-1+z_{2, y}-1\right|_{p}} \begin{cases}\geqslant 3^{k}, & \text { если } p=3, \\
=1, & \text { если } p \neq 3 .\end{cases}
\end{aligned}
$$

Таким образом, решение системы (4) может существовать, только если $p>3$. Если $p>3$, то

$$
\left|\prod_{y \in S(x)}\left(1+z_{1, y}+z_{2, y}\right)\right|_{p}=\left|\prod_{y \in S(x)}\left(3+z_{1, y}-1+z_{2, y}-1\right)\right|_{p}=1 .
$$

Предположим, что $S(x)=\left\{x_{1}, \ldots, x_{k}\right\}$. Учитывая, что $p>3$, из уравнений (4) получим

$$
\begin{aligned}
&\left|z_{1, x}-1\right|_{p}=\left|\lambda \prod_{i=1}^{k} \frac{1+z_{1, y_{i}}}{1+z_{1, y_{i}}+z_{2, y_{i}}}-1\right|_{p}=\left|\lambda \prod_{i=1}^{k}\left(1+z_{1, y_{i}}\right)-\prod_{i=1}^{k}\left(1+z_{1, y_{i}}+z_{2, y_{i}}\right)\right|_{p}= \\
&=\left|(\lambda-1) \prod_{i=1}^{k}\left(1+z_{1, y_{i}}\right)+\prod_{i=1}^{k}\left(2+z_{1, y_{i}}-1\right)-\prod_{i=1}^{k}\left(3+z_{1, y_{i}}-1+z_{2, y_{i}}-1\right)\right|_{p}= \\
&=\mid(\lambda-1) \prod_{i=1}^{k}\left(1+z_{1, y_{i}}\right)+\left(\prod_{i=1}^{k}\left(z_{1, y_{i}}-1\right)-\prod_{i=1}^{k}\left(z_{1, y_{i}}-1+z_{2, y_{i}}-1\right)\right)+ \\
& \\
&+\sum_{j=1}^{k}\left(2 \prod_{\substack{1 \leqslant i \leqslant k \\
i \neq j}}\left(z_{1, y_{i}}-1\right)-3 \prod_{\substack{1 \leqslant i \leqslant j \\
i \neq j}}\left(z_{1, y_{i}}-1+z_{2, y_{i}}-1\right)\right)+\cdots \\
& \cdots+\sum_{i=1}^{k}\left(2^{k-1}\left(z_{1, y_{i}}-1\right)-3^{k-1}\left(z_{1, y_{i}}-1+z_{2, y_{i}}-1\right)\right)+\left.\left(2^{k}-3^{k}\right)\right|_{p} .
\end{aligned}
$$

Используя лемму 2 , получаем, что правая часть равенства (5) меньше $p^{1 /(1-p)}$, если $3^{k}-2^{k}$ делится на $p$, и равна единице в противном случае. 
ТАБЛИЦА 1

\begin{tabular}{|c|c|c|c|c|c|c|c|c|c|c|c|c|}
\hline$k$ & 1 & 2 & 3 & 4 & 5 & 6 & 7 & 8 & 9 & 10 & 11 & 12 \\
\hline$p$ & - & - & - & 5 & - & 7 & - & 5 & - & 11 & 23 & 5,7 \\
\hline
\end{tabular}

Аналогично,

$$
\begin{gathered}
\left|z_{2, x}-1\right|_{p}=\left|\lambda \prod_{i=1}^{k} \frac{1}{1+z_{1, y_{i}}+z_{2, y_{i}}}-1\right|_{p}=\left|(\lambda-1)+1-\prod_{i=1}^{k}\left(1+z_{1, y_{i}}+z_{2, y_{i}}\right)\right|_{p}= \\
=\mid(\lambda-1)-\prod_{i=1}^{k}\left(z_{1, y_{i}}-1+z_{2, y_{i}}-1\right)-3 \sum_{j=1}^{k} \prod_{\substack{1 \leqslant i \leqslant k \\
i \neq j}}\left(z_{1, y_{i}}-1+z_{2, y_{i}}-1\right)-\cdots \\
\cdots-3^{k-1} \sum_{j=1}^{k}\left(z_{1, y_{i}}-1+z_{2, y_{i}}-1\right)-\left.\left(3^{k}-1\right)\right|_{p},
\end{gathered}
$$

и правая часть последнего равенства меньше $p^{1 /(1-p)}$, если $3^{k}-1$ делится на $p$, и равна единице в противном случае.

Таким образом, решение $z_{x}$ принадлежит множеству $\mathcal{E}_{p}^{2}$ в том и только в том случае, когда наибольший общий делитель $\left(2^{k}-1,3^{k}-1\right)$ делится на $p$.

ЗАмЕчАниЕ 1. Условие, что наибольший общий делитель $\left(2^{k}-1,3^{k}-1\right)$ делится на $p$, необходимо для существования $p$-адических мер Гиббса в $G$-модели ТС. Из этого условия для $k \geqslant 1$ можно найти значения простого числа $p$, при которых система уравнений (4) может иметь решение (см. табл. 1 при малых значениях $k$ ).

Теорема 5. Если наибольший общий делитель $\left(2^{k}-1,3^{k}-1\right)$ делится на $р$ и число $k^{2}-k-6$ не делится на $p$, то система уравнений (4) имеет по крайней мере одно решение $z_{x} \in \mathcal{E}_{p}^{2}$ для $x \in V$.

ДокАЗАТЕЛЬство. Проверим, что при удовлетворении условий теоремы система уравнений (4) имеет решение вида $z_{x}=\left(z_{1}, z_{2}\right) \in \mathcal{E}_{p}^{2}$ для $x \in V$. В этом случае из уравнений (4) мы получаем

$$
z_{1}=\lambda\left(\frac{1+z_{1}}{1+z_{1}+z_{2}}\right)^{k}, \quad z_{2}=\lambda\left(\frac{1}{1+z_{1}+z_{2}}\right)^{k} .
$$

Разделим второе уравнение на первое и найдем $z_{2}=z_{1} /\left(1+z_{1}\right)^{k}$. Подставляя это выражение в первое уравнение из (6), мы получаем

$$
z_{1}=\lambda\left(\frac{\left(1+z_{1}\right)^{k+1}}{z_{1}+\left(1+z_{1}\right)^{k+1}}\right)^{k}
$$

Это уравнение можно записать как $F(z)=0$ при

$$
F(z)=z\left(z+(1+z)^{k+1}\right)^{k}-\lambda(1+z)^{k^{2}+k} .
$$


Так как $|\lambda|_{p}=1$, функция $F(z)$ является многочленом с целыми $p$-адическими коэффициентами. Остается проверить остальные условия леммы Гензеля для $F(z)$. Пусть $a_{0}=1$, тогда

$$
\begin{aligned}
F(1) & =\left(1+2^{k+1}\right)^{k}-\lambda 2^{k^{2}+k}=\left(3+2^{k}-1\right)^{k}-1-(\lambda-1) 2^{k^{2}+k}+\left(2^{k^{2}+k}-1\right)= \\
& =\sum_{i=1}^{k} C_{k}^{i} 3^{k-i}\left(2^{k}-1\right)^{i}+3^{k}-1-(\lambda-1) 2^{k^{2}+k}+\left(2^{k}-1\right) \sum_{i=0}^{k} 2^{k i}=0(\bmod p) .
\end{aligned}
$$

Теперь проверим, что если $k^{2}-k-6$ не делится на $p$, то $F^{\prime}(1) \neq 0(\bmod p)$. Имеем

$$
\begin{aligned}
F^{\prime}(1)= & \left(1+2^{k+1}\right)^{k}+k\left(1+2^{k+1}\right)^{k-1}\left(1+(k+1) 2^{k}\right)-\left(k^{2}+k\right) \lambda 2^{k^{2}+k-1}= \\
= & \left(2^{k}-1\right)\left(\sum_{i=1}^{k-1} C_{k-1}^{i} 3^{k-i-1}\left(2^{k}-1\right)^{i-1}\left(\left(2^{k}-1\right)\left(k^{2}+k+1\right)+k^{2}+2 k+3\right)+\right. \\
& \left.+3^{k-1}\left(k^{2}+k+1\right)-\frac{k^{2}+k}{2}\right)+\frac{\left(3^{k}-1\right)\left(k^{2}+2 k+3\right)}{3}- \\
& -(\lambda-1)\left(k^{2}+k\right) 2^{k^{2}+k-1}-\frac{k^{2}-k-6}{6}=k^{2}-k-6(\bmod p) .
\end{aligned}
$$

Таким образом, для функции $F(z)$ выполняются условия леммы Гензеля. Следовательно, существует единственное целое $p$-адическое число $а$ такое, что $F(a)=0$ и $a=a_{0}(\bmod p)$, т. е. уравнение $F(z)=0$ имеет решение $z=a$. При этом $a \in \mathcal{E}_{p}$, так как $a_{0}=1$ и $a \equiv 1(\bmod p)$.

В качестве следствия из этой теоремы мы получаем следующее утверждение.

Теорема 6. Если наибольший общий делитель $\left(2^{k}-1,3^{k}-1\right)$ делится на $р$ и число $k^{2}-k-6$ не делится на $p$, то для $p$-адической $G$-модели ТC существует единственная трансляиионно-инвариантная р-адическая мера Гиббса.

ЗАмечАниЕ 2. Условие, что $k^{2}-k-6$ не делится на $p$, не является необходимым для существования и единственности трансляционно-инвариантной $p$-адической меры Гиббса.

ПримеР 1. Пусть $k=8$ и $p=5$. Заметим, что в этом случае наибольший общий делитель $\left(2^{k}-1,3^{k}-1\right)$ делится на $p$ (см. табл. 1$)$. При этом $k^{2}-k-6$ делится на $p$. Рассмотрим функцию

$$
F(z)=\sum_{i=0}^{7}(1+z)^{9 i}\left(z+(1+z)^{9}\right)^{7-i}
$$

Эта функция является многочленом с целыми $p$-адическими коэффициентами, отсюда, используя лемму Гензеля, мы убеждаемся, что уравнение $F(z)=0$ имеет единственное решение на множестве $\mathcal{E}_{5}$. Обозначим это решение через $z^{*}$. Если $\lambda=z^{*}$, то, как легко видеть, пара значений $\left(\lambda, \lambda /(1+\lambda)^{k}\right)$ является решением системы (6). Таким образом, при $k=8, p=5$ не выполняется условие теоремы 6 , но существует трансляционно-инвариантная $p$-адическая мера Гиббса. 
ТАБЛИЦА 2

\begin{tabular}{|c|c|c|c|c|c|c|c|c|c|c|}
\hline$k$ & 1 & 2 & 3 & 4 & 5 & 6 & 7 & 8 & 9 & 10 \\
\hline$p$ & - & 3 & 7 & 3,5 & 31 & 3,7 & 127 & $3,5,17$ & 7,73 & $3,11,31$ \\
\hline
\end{tabular}

Рассмотрим уравнение

$$
\sum_{i=0}^{k-1}(1+z)^{(k+1) i}\left(z+(1+z)^{k+1}\right)^{k-1-i}=0 .
$$

С помощью леммы Гензеля можно легко проверить, что если наибольший общий делитель $\left(2^{k}-1,3^{k}-1\right)$ делится на $p$ и $k(k-1)$ не делится на $p$, то уравнение $(7)$ имеет решение на $\mathcal{E}_{p}$. В этом случае если $\lambda$ является решением уравнения (7), то $\left(\lambda, \lambda /(1+\lambda)^{k}\right)$ является решением системы уравнений (6). Таким образом, верна следующая теорема.

ТеОрема 7. Пусть наибольший общий делитель $\left(2^{k}-1,3^{k}-1\right)$ делится на $p$ и число $k^{2}-k$ не делится на $p$. Если $\lambda$ является решением уравнения (7), то существует по крайней мере одна транслячионно-инвариантная р-адическая мера Гиббса для $G$-модели ТC.

3.2. Случай $G=$ свисток. В этом случае система уравнений $(3)$ имеет следующий вид:

$$
z_{1, x}=\lambda \prod_{y \in S(x)} \frac{1+z_{2, y}}{1+z_{1, y}}, \quad z_{2, x}=\lambda \prod_{y \in S(x)} \frac{z_{1, y}}{1+z_{1, y}} .
$$

Как и в случае $G=$ ключ, можно доказать следующую теорему.

Теорема 8. Если $2^{k}-1$ не делится на р, то система уравнений (8) не имеет решений на множестве $\mathcal{E}_{p}^{2}$.

ЗАмечАниЕ 3. Условие, что $2^{k}-1$ делится на $p$, является необходимым для существования $p$-адических мер Гиббса для $G$-модели ТС. Для $k \geqslant 1$ можно найти значения простого числа $p$, при которых система уравнений (8) может иметь решение (см. табл. 2 при малых значениях $k$ ).

Пусть $z_{i, x}=z_{i} \in \mathcal{E}_{p}$ при всех $x \in V$. Тогда из уравнений (8) имеем

$$
z_{1}=\lambda\left(\frac{1+z_{2}}{1+z_{1}}\right)^{k}, \quad z_{2}=\lambda\left(\frac{z_{1}}{1+z_{1}}\right)^{k}
$$

УтВЕРЖДЕНИЕ 1. Пусть $2^{k}-1$ делится на р. Справедливы следующие утверждения.

1. Если $\lambda$ является решением уравнения

$$
\lambda\left(\lambda+2^{k}\right)^{k}-2^{k^{2}+k}=0,
$$

то $\left(1, \lambda / 2^{k}\right)$ является решением системы уравнений (9).

2. Если $k+2$ не делится на $p$, то уравнение (10) имеет единственное решение на множестве $\mathcal{E}_{p}$. 
ДокАзАТЕЛьство. Подставляя $z=\left(1, \lambda / 2^{k}\right)$ в $(9)$, получаем уравнение (10). При условии, что $k+2$ не делится на $p$, существование и единственность решения уравнения (10) следует из леммы Гензеля.

Аналогично можно доказать следующее

УтвеРЖДЕНИЕ 2. Пусть $2^{k}-1$ делится на р. Справедливы следующие утверждения.

1. Если $\lambda$ является решением уравнения

$$
(1+\lambda)^{k}-\lambda^{k}=0
$$

то $(\lambda, \lambda)$ является решением системы уравнений (9).

2. Если $k$ не делится на $p$, то уравнение (11) имеет единственное решение на множестве $\mathcal{E}_{p}$.

Теорема 9. Если $2^{k}-1$ делится на $р$ и $k^{2}-2 k-4$ не делится на $p$, то для р-адической $G$-модели ТC существует единственная транслячионно-инвариантная р-адическая мера Гиббса.

ДокАзАТЕЛЬСтво. Из уравнений (9) получаем уравнение

$$
z(1+z)^{k^{2}+k}-\lambda\left((1+z)^{k}+\lambda z^{k}\right)^{k}=0
$$

Если $k^{2}-2 k-4$ не делится на $p$, то функция

$$
F(z)=z(1+z)^{k^{2}+k}-\lambda\left((1+z)^{k}+\lambda z^{k}\right)^{k}
$$

удовлетворяет условию леммы Гензеля. Следовательно, уравнение (12) имеет единственное решение $z^{*} \in \mathcal{E}_{p}$.

ЗАмЕчАниЕ 4. Условие, что $k^{2}-2 k-4$ не делится на $p$, является лишь достаточным для того, чтобы существовала единственная трансляционно-инвариантная $p$ адическая мера Гиббса для $G$-модели ТС.

Пример 2. Пусть $k=16$ и $p=5$. В этом случае условие теоремы 9 не выполняется. Так как $k$ не делится на $p$, в силу утверждения 2 уравнение (11) имеет единственное решение на множестве $\mathcal{E}_{p}$. Предположим, что $\lambda$ является решением уравнения (11). Тогда $z^{*}=(\lambda, \lambda)$ является решением системы уравнений (9) (см. утверждение 2). Таким образом мы показали, что при $k=16, p=5$ существует трансляционно-инвариантная $p$-адическая мера Гиббса.

СлеДСтвиЕ 1. Пусть $p \in\{3,7\}$. Если $2^{k}-1$ делится на $p$, то для $p$-адической $G$-модели TC существует единственная трансляционно-инвариантная р-адическая мера Гиббса.

Доказательство. Пусть $p \in\{3,7\}$. Покажем, что $k^{2}-2 k-4 \neq 0(\bmod p)$ при всех $k \in \mathbb{N}$. Для $p=3$ имеем

$$
k^{2}-2 k-4=\left\{\begin{array}{ll}
1(\bmod 3), & \text { если } k=3 n+1, \\
2(\bmod 3), & \text { если } k=3 n, k=3 n+2,
\end{array} \quad n=0,1,2, \ldots .\right.
$$


Для $p=7$ имеем

$$
k^{2}-2 k-4=\left\{\begin{array}{ll}
2(\bmod 7), & \text { если } k=7 n+1, \\
3(\bmod 7), & \text { если } k=7 n, k=7 n+2, \\
4(\bmod 7), & \text { если } k=7 n+4, k=7 n+5, \\
6(\bmod 7), & \text { если } k=7 n+3, k=7 n+6,
\end{array} \quad n=0,1,2, \ldots\right.
$$

В силу теоремы 9 существует единственная трансляционно-инвариантная $p$-адическая мера Гиббса.

3.3. Случай $G=$ жезл. В этом случае система уравнений (3) имеет следующий вид:

$$
z_{1, x}=\lambda \prod_{y \in S(x)} \frac{1+z_{1, y}}{z_{1, y}+z_{2, y}}, \quad z_{2, x}=\lambda \prod_{y \in S(x)} \frac{1+z_{2, y}}{z_{1, y}+z_{2, y}} .
$$

Теорема 10. Пусть $p=2$. Если $k$ делится на 4 , то система уравнений (13) имеет по крайней мере одно решение $z_{x} \in \mathcal{E}_{p}^{2}$ для $x \in V$.

ДоказАтельство. Предположим, что $z_{i, x}=z, i=1,2$, при всех $x \in V$. Тогда

$$
z=\lambda\left(\frac{1+z}{2 z}\right)^{k}
$$

Положим $F(z)=\lambda((1+z) / 2 z)^{k}$. Пусть $z \in \mathcal{E}_{2}$. Имеем

$$
\begin{aligned}
|F(z)|_{2}= & \left|\lambda\left(\frac{1+z}{2 z}\right)^{k}\right|_{2}=2^{k}\left|(2+z-1)^{k}\right|_{2}=2^{k}\left|2^{k}+\sum_{i=1}^{k} C_{k}^{i} 2^{k-i}(z-1)^{i}\right|_{2}=1, \\
|F(z)-1|_{2}= & \left|(\lambda-1)\left(\frac{1+z}{2 z}\right)^{k}+\left(\frac{1+z}{2 z}\right)^{k}-1\right|_{2}= \\
= & \mid(\lambda-1)\left(\frac{1+z}{2 z}\right)^{k}-\frac{z-1}{z}\left(\frac{k}{2}+\sum_{m=0}^{k}\left(\sum_{i=1}^{m} C_{m}^{i} 2^{-i-1}(z-1)^{i}+\right.\right. \\
& +\sum_{j=1}^{k-m-1} C_{k-m-1}^{j} \frac{(z-1)^{j}}{2}+ \\
& \left.\left.+\sum_{i=1}^{m} C_{m}^{i} 2^{m-k-i}(z-1)^{i} \sum_{j=1}^{k-1-m} C_{k-1-m^{j}}^{j} 2^{-m-1}(z-1)^{j}\right)\right)\left.\right|_{2} \leqslant \frac{1}{4} .
\end{aligned}
$$

Таким образом мы показали, что $F(z)=\lambda((1+z) / 2 z)^{k}$ есть отображение из $\mathcal{E}_{2}$ в $\mathcal{E}_{2}$. Для точки $z \in \mathcal{E}_{2}$ имеем

$$
\left|F^{\prime}(z)\right|_{2}=\left|-\frac{k \lambda(1+z)^{k-1}}{2^{k} z^{k+1}}\right|_{2}=\left|\frac{k}{2}\right|_{2} \leqslant \frac{1}{2} .
$$

Следовательно [20], для всех $a, b \in \mathcal{E}_{2}$

$$
|F(a)-F(b)|_{2} \leqslant \frac{1}{2}|a-b|_{2} .
$$

Так как $F$ является сжимающим отображением на замкнутом множестве $\mathcal{E}_{2}$, уравнение (14) имеет единственное решение $z^{*} \in \mathcal{E}_{2}$. 
Как следствие этой теоремы получаем следующий результат.

Теорема 11. Пусть $p=2$. Если $k$ делится на 4, то для р-адической $G$-модели ТС существует единственная трансляционно-инвариантная р-адическая мера Гиббса.

Предположим, что $z_{i}=z_{i, x}, i=1,2$, при всех $x \in V$. Тогда система уравнений (13) имеет следующий вид:

$$
z_{1}=\lambda\left(\frac{1+z_{1}}{z_{1}+z_{2}}\right)^{k}, \quad z_{2}=\lambda\left(\frac{1+z_{2}}{z_{1}+z_{2}}\right)^{k} .
$$

Вычитая второе уравнение системы (15) из первого, получаем

$$
\left(z_{1}-z_{2}\right)\left(1-\lambda \frac{\left(1+z_{1}\right)^{k-1}+\cdots+\left(1+z_{2}\right)^{k-1}}{\left(z_{1}+z_{2}\right)^{k}}\right)=0 .
$$

Таким образом, либо $z_{1}=z_{2}$, либо

$$
\left(z_{1}+z_{2}\right)^{k}=\lambda\left(\left(1+z_{1}\right)^{k-1}+\cdots+\left(1+z_{2}\right)^{k-1}\right) \quad \text { при } z_{1} \neq z_{2} .
$$

Теорема 12. Пусть $p \neq 2$. Если $k+2$ не делится на $p$, то система уравнений (13) имеет по крайней мере одно решение $z_{x} \in \mathcal{E}_{p}^{2}$ для $x \in V$.

ДокАзАТЕЛьство. Достаточно показать, что при выполнении условия теоремы уравнение $(14)$ имеет решение на $\mathcal{E}_{p}$. Используя лемму Гензеля для функции

$$
F(z)=2^{k} z^{k+1}-\lambda(1+z)^{k}
$$

можно убедиться, что уравнение (14) имеет единственное решение на $\mathcal{E}_{p}$.

Теорема 13. Пусть $p \neq 2$. Если число $k^{2}-4$ не делится на $p$, то для $p$-адической $G$-модели TC существует единственная транслящионно-инвариантная р-адическая мера Гиббса.

ДокАЗАтельство. Пусть $p \neq 2$. Так как $k+2$ не делится на $p$, уравнение (14) имеет единственное решение (см. доказательство теоремы 12). Надо показать, что если $k-2$ не делится на $p$, то уравнение (16) не имеет решения на множестве $\mathcal{E}_{p}^{2}$. Вычитая $2^{k}$ из обеих частей уравнения (16), получаем

$$
\left(z_{1}+z_{2}\right)^{k}-2^{k}=\left(z_{1}-1+z_{2}-1\right)\left(\left(z_{1}+z_{2}\right)^{k-1}+\cdots+2^{k-1}\right)=0(\bmod p)
$$

и

$$
\begin{aligned}
& \lambda \sum_{i=0}^{k-1}\left(1+z_{1}\right)^{i}\left(1+z_{2}\right)^{k-1-i}-2^{k}=(\lambda-1) \sum_{i=0}^{k-1}\left(1+z_{1}\right)^{i}\left(1+z_{2}\right)^{k-1-i}+ \\
& \quad+\sum_{i=0}^{k-1}\left(2+z_{1}-1\right)^{i}\left(2+z_{2}-1\right)^{k-1-i}-2^{k}=2^{k-1}(k-2)(\bmod p)=k-2(\bmod p) .
\end{aligned}
$$

Отсюда следует, что уравнение (16) не имеет решения на множестве $\mathcal{E}_{p}^{2}$, если $k-2$ не делится на $p$. 
ЗАмЕЧАниЕ 5. Условие теоремы 13 не является необходимым для единственности трансляционно-инвариантной меры Гиббса.

Пример 3. Пусть $k=2, p=3$ и $\lambda=1$. Покажем, что в этом случае для $G$-модели ТC существует единственная трансляционно-инвариантная $p$-адическая мера Гиббса. Уравнение (14) имеет единственное решение $z=1$, так как $k+2$ не делится на $p$ (см. теорему 12). Следовательно, $z^{*}=(1,1)$ является единственным решением системы (15) на множестве $\left\{z=\left(z_{1}, z_{2}\right) \in \mathcal{E}_{3}^{2}: z_{1}=z_{2}\right\}$. При $k=2$ уравнение (16) имеет следующий вид:

$$
\left(z_{1}+z_{2}\right)^{2}-\left(z_{1}+z_{2}\right)-2=0 .
$$

Отсюда $z_{1}+z_{2}=-1$ или $z_{1}+z_{2}=2$. Нетрудно убедиться, что в этих случаях система уравнений (15) не имеет решения на множестве $\left\{z=\left(z_{1}, z_{2}\right) \in \mathcal{E}_{3}^{2}: z_{1} \neq z_{2}\right\}$. Таким образом, при $k=2, p=3$ система уравнений (15) имеет единственное решение на множестве $\mathcal{E}_{3}^{2}$. Это означает, что в данном случае существует единственная трансляционно-инвариантная $p$-адическая мера Гиббса для $G$-модели ТС.

Tеорема 14. Пусть $k=2, p=3$. Если $\lambda \in\left\{x \in \mathbb{Q}_{3}:|x-28| \leqslant 1 / 81\right\}$, mo для р-адической $G$-модели ТC существуют три транслячионно-инвариантные $p$ адические меры Гиббса.

ДокАЗАтЕЛьСтво. Пусть $k=2, p=3$. В силу теоремы 12 уравнение (14) имеет единственное решение $z_{1}^{*} \in \mathcal{E}_{p}$. Следовательно, $z^{*}=\left(z_{1}^{*}, z_{1}^{*}\right) \in \mathcal{E}_{p}^{2}$ есть одно из решений уравнений (15).

Покажем, что система уравнений (15) имеет еще два решения $z^{-}=\left(z_{1}^{-}, z_{2}^{-}\right)$ и $z^{+}=\left(z_{1}^{+}, z_{2}^{+}\right)$, где $z_{1}^{-} \neq z_{2}^{-}$и $z_{1}^{+} \neq z_{2}^{+}$. Из соотношения (16) получаем

$$
z_{1}+z_{2}=\frac{\lambda \pm \sqrt{\lambda(\lambda+8)}}{2} .
$$

Подставляя это равенство в (15), имеем

$$
z=\left(\frac{2(1+z)}{\sqrt{\lambda} \pm \sqrt{\lambda+8}}\right)^{2}
$$

Найдем решения этого квадратного относительно z уравнения:

$$
\begin{aligned}
& a^{ \pm}=\frac{(\sqrt{\lambda}+\sqrt{\lambda+8})(2 \sqrt{\lambda} \pm \sqrt{2(\lambda-4+\sqrt{\lambda(\lambda+8)})})}{8}, \\
& b^{ \pm}=\frac{(\sqrt{\lambda}-\sqrt{\lambda+8})(2 \sqrt{\lambda} \pm \sqrt{2(\lambda-4-\sqrt{\lambda(\lambda+8)})})}{8} .
\end{aligned}
$$

Теперь мы должны проверить, что $\sqrt{\lambda}, \sqrt{\lambda+8}$ и $\sqrt{2(\lambda-4 \pm \sqrt{\lambda(\lambda+8)})}$ существуют в $\mathbb{Q}_{3}$ и что $a^{ \pm}, b^{ \pm} \in \mathcal{E}_{3}$. Из условия $\lambda \in\left\{x \in \mathbb{Q}_{3}:|x-28|_{3} \leqslant 1 / 81\right\}$ имеем следующее представление:

$$
\lambda=1+1 \cdot 3^{3}+\lambda_{4} \cdot 3^{4}+\lambda_{5} \cdot 3^{5}+\cdots
$$


Легко видеть, что $\lambda$ и $\lambda+8$ удовлетворяют условиям теоремы 1 . Следовательно, $\sqrt{\lambda}$ и $\sqrt{\lambda+8}$ существуют в $\mathbb{Q}_{3}$. Из представления (19) имеем

$$
\sqrt{\lambda(\lambda+8)}=3\left(1+2 \cdot 3+a_{2} \cdot 3^{2}+a_{3} \cdot 3^{3}+\cdots\right)
$$

Отсюда

$$
\begin{aligned}
2(\lambda-4 & +\sqrt{\lambda(\lambda+8)})= \\
& =2\left(2 \cdot 3^{2}+\left(1+a_{2}\right) \cdot 3^{3}+\left(\lambda_{4}+a_{3}\right) \cdot 3^{4}+\left(\lambda_{5}+a_{4}\right) \cdot 3^{5}+\cdots\right)= \\
& =3^{2}\left(1+\lambda_{1}^{\prime} \cdot 3+\lambda_{2}^{\prime} \cdot 3^{2}+\cdots\right) .
\end{aligned}
$$

В силу теоремы $1 \sqrt{2(\lambda-4+\sqrt{\lambda(\lambda+8)})}$ существует в $\mathbb{Q}_{3}$.

Положим

$$
c^{2}=2(\lambda-4+\sqrt{\lambda(\lambda+8)}), \quad d=2(\lambda-4-\sqrt{\lambda(\lambda+8)}) .
$$

Перемножая эти равенства, получим $64(1-\lambda)=c^{2} d$. Поскольку $\sqrt{-3} \notin \mathbb{Q}_{3}$, отсюда следует, что $\sqrt{1-\lambda} \notin \mathbb{Q}_{3}$. Следовательно, $\sqrt{d} \notin \mathbb{Q}_{3}$. Это доказывает, что значения (18) не являются решением системы уравнений (15).

Теперь проверим, что $a^{ \pm} \in \mathcal{E}_{3}$. Из соотношений (19)-(21) имеем

$$
|\sqrt{\lambda}|_{3}=1, \quad|\sqrt{\lambda+8}|_{3}<1, \quad|\sqrt{2(\lambda-4+\sqrt{\lambda(\lambda+8)})}|_{3}<1 .
$$

Следовательно, $\left|a^{ \pm}\right|_{3}=1$ и $\left|a^{ \pm}-1\right|_{3}<1 / 3$. Положим $z_{1}^{-}=a^{-}, z_{2}^{-}=a^{+}$и $z_{1}^{+}=a^{+}$, $z_{2}^{+}=a^{-}$. В результате мы получили в точности три решения:

$$
z^{*}=\left(z_{1}^{*}, z_{1}^{*}\right), \quad z^{-}=\left(z_{1}^{-}, z_{2}^{-}\right), \quad z^{+}=\left(z_{1}^{+}, z_{2}^{+}\right) .
$$

Благодарности. Автор благодарен профессору У. А. Розикову за полезные советы и ряд замечаний.

\section{Список литературы}

[1] J. B. Martin, U. A. Rozikov, Yu. M. Suhov, J. Nonlin. Math. Phys, 12:3 (2005), 432-448.

[2] У. А. Розиков, Ш. А. Шоюсупов, ТМФ, 156:3 (2008), 412-424.

[3] D. Gandolfo, U. A. Rozikov, J. Ruiz, Markov Process. Related Fields, 18:4 (2012), 701-720.

[4] P. M. Bleher, J. Ruiz, V. A. Zagrebnov, J. Statist. Phys., 79:1-2 (1995), 473-482.

[5] Н. Н. Ганиходжаев, ТМФ, 85:2 (1990), 163-175.

[6] H.-O. Georgii, Gibbs Measures and Phase Transitions, de Gruyter Studies in Mathematics, 9, Walter de Gruyter, Berlin, 1988.

[7] Y. M. Suhov, U. A. Rozikov, Queueing Syst., 46:1-2 (2004), 197-212.

[8] A. Yu. Khrennikov, Non-Archimedean Analysis: Quantum Paradoxes, Dynamical Systems and Biological Models, Mathematics and its Applications, 427, Kluwer, Dordrecht, 1997.

[9] E. Marinari, G. Parisi, Phys. Lett. B, 203:1-2 (1988), 52-54.

[10] В. С. Владимиров, И. В. Волович, Е. И. Зеленов, р-Адический анализ и математическая физика, Наука, М., 1994.

[11] A. C. M. van Rooij, Non-Archimedean Functional Analysis, Monographs and Textbooks in Pure and Applied Mathematics, 51, M. Dekker, New York, 1978.

[12] S. Albeverio, W. Karwowski, Stoch. Proc. Appl., 53:1 (1994), 1-22. 
[13] S. Albeverio, X. Zhao, Ann. Probab., 28:4 (2000), 1680-1710.

[14] K. Yasuda, Osaka J. Math., 37:4 (2000), 967-985.

[15] У. А. Розиков, О. Н. Хакимов, ТМФ, 175:1 (2013), 84-92.

[16] Н. Н. Ганиходжаев, Ф. М. Мухаммедов, У. А. Розиков, Узб. матем. журн., 4 (1998), 23-29.

[17] G. R. Brightwell, P. Winkler, J. Combin. Theory Ser. B, 77:2 (1999), 221-262.

[18] Ф.Ф. Тураев, Узб. матем. журн., 2 (2013), 21-29.

[19] Н. Коблиц, -Адические числа, р-адический анализ и дзета-функции, Мир, М., 1982.

[20] W. H. Schikhof, Ultrametric Calculus. An introduction to p-adic analysis, Cambridge Studies in Advanced Mathematics, 4, Cambridge Univ. Press, Cambridge, 1984.

[21] F. M. Mukhamedov, U. A. Rozikov, Indag. Math. (N. S.), 15:4 (2004), 85-99. 